\title{
Insufficient sleep is prevalent among migraineurs: a population-based study
}

\author{
Jiyoung Kim ${ }^{1}$, Soo-Jin Cho ${ }^{2}$, Won-Joo Kim ${ }^{3}$, Kwang Ik Yang ${ }^{4}$, Chang-Ho Yun ${ }^{5}$ and Min Kyung Chu ${ }^{6 *}$
}

\begin{abstract}
Background: Sleep disorder and sleep complaints are common in subjects with migraine. Although the association between sleep disorders and migraine has been reported, the association between perceived insufficient sleep and migraine has rarely reported. The aim of this study is to evaluate the association between insufficient sleep and migraine using the data of the Korean Headache-Sleep Study (KHSS).

Methods: The KHSS is a nation-wide cross-sectional population-based survey regarding headache and sleep for Korean adults aged 19 to 69 years. A difference of one hour or more between sleep need and average sleep time indicated insufficient sleep.

Results: Of 2,695 participants, 727 (27.0\%) individuals were classified as having insufficient sleep. The prevalence of insufficient sleep among individuals with migraine (45.5\%) was significantly higher compared to that among individuals with non-migraine headache $(32.9 \%, p=0.004)$ or among non-headache $(20.4 \%, p<0.001)$. Average sleep time did not differ among migraine, non-migraine headache, and non-headache groups (7.3 \pm 1.2 vs. $7.2 \pm 1.2$ vs. $7.3 \pm 1.4, p=0.207)$. Multivariable logistic regression analyses demonstrated that migraine had an increased odds ratio (OR) for insufficient sleep after adjusting for sociodemographic variables, short sleep time, insomnia, poor sleep quality, anxiety, and depression ( $\mathrm{OR}=1.8,95 \%$ confidence interval $[\mathrm{Cl}]=1.2-2.7, p=0.002)$.
\end{abstract}

Conclusions: The prevalence of insufficient sleep was significantly higher among migraineurs compared to that in non-migraine headache or non-headache group.

Keywords: Migraine, Sleep, Sleep deprivation, Sleep time, Epidemiology

\section{Background}

Migraine and sleep disturbance are common complaints in the general population. People who suffer from migraine and sleep disturbance often experience disability and decreased quality of life. Therefore, both conditions impose significant amounts of personal and social burden.

Epidemiological and clinic-based studies have demonstrated a close association between migraine and sleep disturbance. They usually occur in the same individuals $[1,2]$. Migraine and insomnia have bidirectional comorbidity, suggesting that they share common mechanisms [3]. Lack of sleep and excessive sleep often trigger migraine attacks $[4,5]$. Snoring is a risk factor for transformation from episodic migraine to chronic migraine

\footnotetext{
* Correspondence: chumk@hallym.ac.kr

${ }^{6}$ Department of Neurology, Kangnam Sacred Heart Hospital, Hallym

University College of Medicine, Seoul, South Korea

Full list of author information is available at the end of the article
}

[6]. Migraineurs report poor sleep quality and daytime tiredness more often than non-migraineurs [7, 8]. Moreover, non-headache symptoms such as tiredness, weary, and yawning have been frequently reported in migraineurs before the headache attack $[9,10]$. These symptoms are associated with insufficient sleep.

Adequate or sufficient sleep has been considered as an essential part of optimal health. Individuals with insufficient sleep have higher risk of adverse health outcomes such as hypertension, heart disease, diabetes, depression, obesity, industrial accidents, and occupational errors [11-13]. However, insufficient sleep is a common problem in the general population $[14,15]$. It is usually associated with sleep disturbance such as insomnia, excessive daytime sleepiness, and late chronotype [14].

Currently, information on the association between migraine and insufficient sleep is limited. The Korean Headache-Sleep Study (KHSS) is a nation-wide, cross- 
sectional study regarding headache and sleep. It provides an opportunity to assess the association between insufficient sleep and migraine. The objectives of the present study were: 1) to determine the prevalence of insufficient sleep and migraine in a general population-based sample; 2) to examine the average sleep time and sleep need among individuals with migraine, non-migraine headache, and non-headache; and 3) to assess the association between insufficient sleep and migraine using the data of KHSS.

\section{Methods}

\section{Study population and survey process}

The KHSS is a nation-wide, cross-sectional survey regarding headache and sleep among Korean adults aged 19 to 69 years. It also included items regarding symptoms of anxiety and depression. The study design, methods, and process were described in details previously [16]. Briefly, we used a 2-stage clustered random sampling method for all Korean territories except Jejudo. It sampled participants proportionally by population distribution. The survey was conducted by door-to-door visit and face-to-face interview by interviewers using a questionnaire. All interviewers were employees of Gallup Korea. They had previous experience of social survey. Data collection of the KHSS was performed from November 2011 to January 2012. The KHSS was approved by the Institutional Review Board and ethics committee of Hallym University Sacred Heart Hospital (IRB No. 2011-I077). Written informed consent was obtained from all participants.

\section{Migraine assessment}

Diagnosis of migraine was based on criteria $A$ to $D$ for migraine without aura (code 1.1) (A, 5 or more attacks in a lifetime; B, attack duration of $4-72 \mathrm{~h}$; $\mathrm{C}$, any 2 of the 4 typical headache characteristics [i.e., unilateral pain, pulsating quality, moderate-to-severe pain intensity, and aggravation by routine physical activity]; and D, attacks associated with at least one of the following: nausea, vomiting, or both photophobia and phonophobia) in the second edition of the international classification of headache disorders (ICHD-2) [17]. If a participant reported an experience of headache during the previous year and her/his most severe type headache met all the criteria, she/he was classified as having migraine. We did not investigate the presence of aura because it was difficult to document it in epidemiological study using a questionnaire [18]. Accordingly, participants who were classified as having migraine might have either migraine with aura (code 1.2) or migraine without aura (code 1.1) in the present study. Our survey method has been reported to have a sensitivity of $75.0 \%$ and a specificity of $88.2 \%$ [19].

\section{Non-migraine headache assessment}

If a participant who was not diagnosed as having migraine responded positively to the question of 'In the past year, have you had at least 1 headache lasting more than 1 min?', she or he was classified as having nonmigraine headaches.

\section{Average sleep time and short sleep time assessment}

We investigated usual sleep time on workdays and free days. Average sleep time was calculated as (weekday sleep time $\times 5+$ weekend sleep time $\times 2$ )/7. If a participant's average sleep time was $\leq 6 \mathrm{~h}$ in a day, she/he was classified as having short sleep time.

\section{Sleep need and insufficient sleep assessment}

We used question "How long do you want to sleep in a day?" to assess sleep need. A difference of one hour or more between sleep need and average sleep time was used to indicate insufficient sleep [14].

\section{Insomnia and poor sleep quality assessment}

We used the Insomnia Severity Index (ISI) to investigate insomnia symptom. ISI is a brief screening questionnaire to measure of insomnia severity. If a participant's ISI score was 10 or more, she or he was classified as having insomnia [20].

The Pittsburgh Sleep Quality Index (PSQI) was used for assessing poor sleep quality. If an individual's PSQI score was 6 or more, she/he was classified as having poor sleep quality.

\section{Anxiety and depression assessment}

We used the Goldberg Anxiety Scale (GAS) to investigate anxiety among participants. This comprises four screening items and five supplementary items [21]. Individuals who gave positive answers to two or more screening items and five or more of all scale items were diagnosed with anxiety. The Korean version of the scale has a sensitivity of $82.0 \%$ and a specificity of $94.4 \%$ for diagnosing anxiety [22]. It has good correlations with the State-Trait Anxiety Inventory, a highly valid tool for assessing anxiety [23].

The Patient Health Questionnaire-9 (PHQ-9) was used to diagnose depression in the present study [24]. Participants who had scores of 10 or more on this measure were considered as having depression. The Korean PHQ-9 has a sensitivity of $81.1 \%$ and a specificity of $89.9 \%$ for diagnosing depression [25].

\section{Analyses}

Kolmogorov-Smirnov test was used to evaluate the normality of the distribution. After normality was confirmed, Student's $t$-test or Analysis of Variance was used to compare continuous variables. Post hoc analyses were 
performed using Turkey's method. Categorical variables were compared using Chi-square test. Significance level was set at $p<0.05$ for all analyses. Statistical analyses were performed using the Statistical Package for Social Sciences 22.0 (SPSS 22.0; IBM, Armonk, NY, USA).

We evaluated odds ratios (ORs) with 95\% confidence interval (CI) for the occurrence of insufficient sleep through univariable and multivariable logistic regression analyses. In univariable analyses, we modelled the ORs for sleep insufficiency without adjusting for covariates. In multivariable analyses, we used 4 models. In Model 1, adjustment was conducted for sociodemographic variables (age, gender, size of residential area, educational level and monthly income level). Model 2 incorporated short sleep time ( $\leq 6 \mathrm{~h}$ in average sleep time), insomnia, and poor sleep quality (PSQI) to Model 1. Model 3 included anxiety (GAS) and depression (PHQ-9) to Model 1. The final model, Model 4, incorporated sociodemographic variables, short sleep time, insomnia, poor sleep quality, anxiety, and depression.

\section{Results}

\section{Survey}

Interviewers approached a total of 7,430 individuals. Of them, 2,695 subjects completed the survey (cooperation rate: $36.3 \%$, Fig. 1). The distribution of age, gender, size of residential area, educational level or monthly income level of our sample was not significantly different from that of the general population of Korea (Table 1).

\section{Prevalence of migraine, non-migraine headache, and insufficient sleep}

Of the 2,695 participants, 143 (5.3\%) subjects were classified as having migraine while 1,130 (41.9\%) individuals and $1,422(52.8 \%)$ individuals were classified as non- migraine headache and non-headache, respectively. Seven hundred and twenty-seven (27.0\%) individuals answered that they needed more than $1 \mathrm{~h}$ of sleep compared to their average sleep time. Therefore, they were classified as having insufficient sleep (Table 1).

\section{Average sleep time, short sleep time, and sleep need}

The average sleep time of all participants was $7.3 \pm 1.2 \mathrm{~h}$ in a day. The average sleep time was not significantly different among individuals with migraine, those with non-migraine headache, and non-headache $(7.3 \pm 1.4$ vs. $7.2 \pm 1.2$ vs. $7.3 \pm 1.2 \mathrm{~h}, p=0.207)$. Four hundred and sixty-nine $(17.4 \%, 95 \% \mathrm{CI}=16.0 \%-18.8 \%)$ individuals were classified as having short sleep time. The prevalence of short sleep time was not significantly different among individuals with migraine, those with nonmigraine headache, and the non-headache $(18.2 \%$ vs. $19.1 \%$ vs. $16.0 \%, p=0.110$ ).

Sleep need of migraineurs $(8.4 \pm 1.5 \mathrm{~h})$ was significantly longer compared to sleep need of those with nonmigraine $(8.0 \pm 1.3 \mathrm{~h}, p<0.001)$ and that of nonheadache individuals $(7.8 \pm 1.3 \mathrm{~h}, p<0.001)$. Among individuals with non-migraine headache, sleep need was longer $(p<0.001)$ than that of individuals with nonheadache (Table 2).

\section{Insomnia and poor sleep quality}

Among 2,695 participants, 290 (10.8\%, 95\% CI $=9.6 \%$ - 11.9\%) and 715 (26.5\%, 95\% CI $=24.8 \%$ - 28.2\%) individuals were classified as having insomnia and poor sleep quality, respectively. Insomnia was more prevalent in individuals with migraine $(25.9 \%, 95 \%$ CI $=18.9 \%-32.9 \%, p<0.001)$ or with non-migraine headache $(15.1 \%, 95 \% \mathrm{CI}=12.8 \%-17.5 \%, p<0.001)$ compared to that in non-headache $(5.8 \%, 95 \% \mathrm{CI}=$

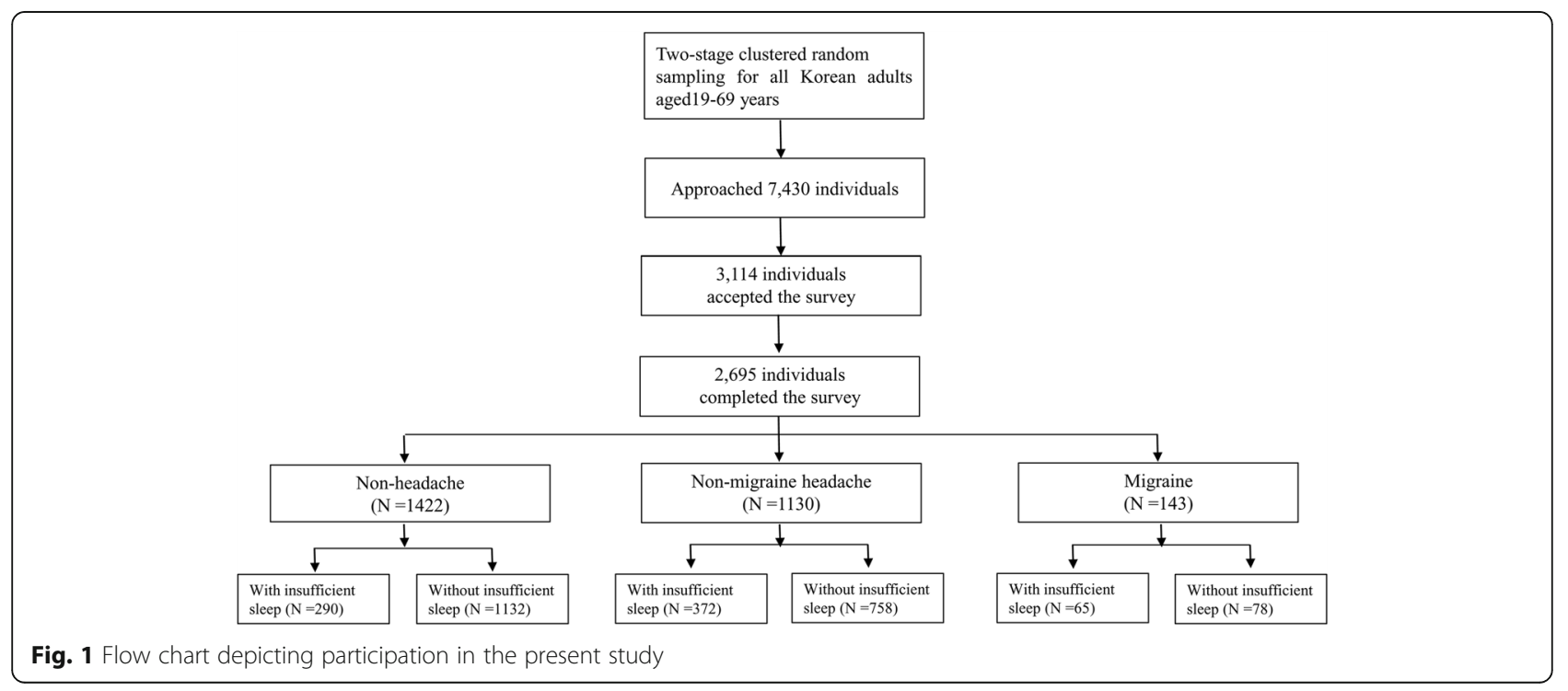


Table 1 Sociodemographic characteristics of survey participants, total Korean population, and cases identified as having migraine, non-migraine headache, or insufficient sleep

\begin{tabular}{|c|c|c|c|c|c|c|}
\hline & $\begin{array}{l}\text { Survey participants } \\
N(\%)\end{array}$ & $\begin{array}{l}\text { Total population } \\
N(\%)\end{array}$ & $P$ & $\begin{array}{l}\text { Migraine } \\
\text { N, \% (95\% Cl) }\end{array}$ & $\begin{array}{l}\text { Non-migraine headache } \\
\text { N, \% (95\% Cl) }\end{array}$ & $\begin{array}{l}\text { Insufficient sleep } \\
N, \%(95 \% \mathrm{Cl})\end{array}$ \\
\hline \multicolumn{7}{|l|}{ Gender } \\
\hline \multirow{2}{*}{$\begin{array}{l}\text { Men } \\
\text { Women }\end{array}$} & $1,345(49.3)$ & $17,584,365(50.6)$ & \multirow[t]{2}{*}{$0.854^{\mathrm{a}}$} & $36,2.7(1.8-3.5)$ & $471,35.0(32.4-37.6)$ & $329,24.5(22.2-26.8)$ \\
\hline & $1,350(50.7)$ & $17,198,350(49.4)$ & & $107,7.9(6.5-9.4)$ & $659,49.0(46.2-51.5)$ & $398,29.5(27.0-31.9)$ \\
\hline \multicolumn{7}{|l|}{ Age } \\
\hline $19-29$ & $542(20.5)$ & $7,717,947(22.2)$ & \multirow[t]{5}{*}{$0.917^{\mathrm{a}}$} & $25,4.5(2.7-6.2)$ & $231,42.6(38.4-46.8)$ & $147,27.1(23.4-30.9)$ \\
\hline $30-39$ & $604(21.9)$ & $8,349,487(24.0)$ & & $42,7.0(4.9-9.1)$ & $269,44.5(40.6-48.5)$ & $174,28.8(25.1-32.4)$ \\
\hline $40-49$ & $611(23.1)$ & $8,613,110(24.8)$ & & $39,6.5(4.5-8.4)$ & $277,45.3(41.4-49.3)$ & $186,30.4(26.7-34.1)$ \\
\hline $50-59$ & $529(18.9)$ & $6,167,505$ (17.7) & & $22,4.1(2.4-5.9)$ & $204,38.6(34.4-42.7)$ & $140,26.5(22.7-30.2)$ \\
\hline $60-69$ & $409(15.6)$ & $3,934,666(11.3)$ & & $15,3.9(2.0-5.7)$ & $149,36.4(31.7-41.1)$ & $80,20.0(15.7-23.4)$ \\
\hline \multicolumn{7}{|l|}{ Size of residential area } \\
\hline Large city & $1,248(46.3)$ & $16,776,771(48.2)$ & \multirow[t]{3}{*}{$0.921^{\mathrm{a}}$} & $76,6.1(4.8-7.5)$ & $525,42.1(39.3-44.8)$ & $334,26.8(24.3-29.2)$ \\
\hline Medium-to-small city & $1186(44.0)$ & $15,164,345(43.6)$ & & $48,4.0(2.9-5.2)$ & $488,41.1(38.3-43.9)$ & $327,27.6(25.0-30.1)$ \\
\hline Rural area & $261(9.7)$ & $2,841,599(8.2)$ & & $19,7.4(4.2-10.6)$ & $117,44.8(38.8-50.9)$ & $66,25.3(20.0-30.6)$ \\
\hline \multicolumn{7}{|l|}{ Education level } \\
\hline Middle school or less & $393(14.9)$ & 6,608,716 (19.0) & \multirow[t]{4}{*}{$0.752^{a}$} & $22,5.5(4.2-7.7)$ & $165,42.0(37.1-46.9)$ & $90,22.9(18.7-27.1)$ \\
\hline High school & $1,208(44.5)$ & $15,234,829(43.8)$ & & $60,5.0(3.8-6.3)$ & $502,41.6(38.8-44.3)$ & $345,28.6(26.0-31.1)$ \\
\hline College or more & 1,068 (39.6) & $12,939,170(37.2)$ & & $60,5.6(4.3-7.0)$ & $457,42.7(39.8-45.8)$ & $286,26.7(24.1-29.4)$ \\
\hline Not responded & $26(1.0)$ & & & 1, $3.8(0.0-11.8)$ & $6,5.3(0.3-9.6)$ & $6,23.1(5.7-40.4)$ \\
\hline \multicolumn{7}{|c|}{ Monthly income (Korean Won ${ }^{b}$ ) } \\
\hline$<2,000,000$ & $403(14.8)$ & $4,452,188(12.8)$ & \multirow[t]{4}{*}{$0.503^{\mathrm{a}}$} & $21,14.7(9.1-20.3)$ & $169,15.0(13.1-17.1)$ & $86,11.8(9.6-14.3)$ \\
\hline $2,000,000-4,999,999$ & $1,813(67.3)$ & $24,417,466(70.2)$ & & $96,67.1(59.4-74.8)$ & $751,66.5(63.8-69.1)$ & $499,68.6(65.2-71.9)$ \\
\hline$\geq 5000000$ & $422(15.7)$ & $5,913,062(17.0)$ & & $22,15.4(9.8-21.7)$ & $186,16.5(14.3-18.7)$ & $124,17.1(14.3-19.9)$ \\
\hline Not responded & $57(2.1)$ & & & $4,2.8(0.7-5.6)$ & $24,2.1(1.3-3.0)$ & $18,2.5(1.4-3.7)$ \\
\hline Total & $2695(100.0)$ & $34,782,715$ (100.0) & & $143,5.3(4.5-6.2)$ & $1130,41.9(40.0-43.8)$ & $727,27.0(25.3-28.7)$ \\
\hline
\end{tabular}

Cl Confidence Interval

${ }^{a}$ Comparison of gender, age group, size of residential area, educational level and monthly income level distributions between the sample in the present study and the total population of Korea

${ }^{\mathrm{b}} 1 \mathrm{USD}=1,123$ Korean Won (February 1, 2012)

$4.6 \%-6.9 \%)$. Poor sleep quality was more prevalent in individuals with migraine $(47.6 \%, 95 \% \mathrm{CI}=39.3 \%-$ $55.8 \%, p<0.001)$ or with non-migraine headache (30.9\%, 95\% CI $=28.2 \%-33.6 \%, p<0.001)$ compared to that in individuals with non-headache $(21.0 \%, 95 \%$ $\mathrm{CI}=18.8 \%-23.1 \%)$. Moreover, the prevalence of poor sleep quality in individuals with migraine was significantly higher compared to that in individuals with non-migraine headache $(p=0.037)$.

\section{Insufficient sleep and migraine}

Among the 143 migraineurs, 65 (45.5\%, 95\% CI = 37.2\% - 53.7\%) individuals were classified as having insufficient sleep. Among the 1,130 individuals with non-migraine headache, $372(32.9 \%, 95 \% \mathrm{CI}=30.2 \%-35.7 \%)$ had insufficient sleep. Among the 1,422 individuals with non-headache, $290(20.4 \%, 95 \%$ CI $=18.3 \%-22.5 \%)$ individuals were found to have insufficient sleep. The prevalence of insufficient sleep was significantly $(p=$

Table 2 Average sleep time and sleep need of individuals with migraine, non-migraine, or non-headache

\begin{tabular}{|c|c|c|c|c|c|}
\hline & Non-headache (1) & Non-migraine headache (2) & Migraine (3) & $P$-value & Post-hoc analysis \\
\hline Average sleep time, hours & $7.3 \pm 1.2$ & $7.2 \pm 1.2$ & $7.3 \pm 1.4$ & 0.207 & \\
\hline \multirow[t]{3}{*}{ Sleep need, hours } & $7.8 \pm 1.3$ & $8.0 \pm 1.3$ & $8.4 \pm 1.5$ & $<0.001$ & (1) vs. (2) $<0.001$ \\
\hline & & & & & (1) vs. (3) $<0.001$ \\
\hline & & & & & (2) vs. (3) $<0.001$ \\
\hline
\end{tabular}


0.004) higher among individuals with migraine compared to that in individuals with non-migraine headache. Additionally, individuals with non-migraine headache had higher $(p<0.001)$ prevalence of insufficient sleep compared to those with non-headache (Fig. 2).

Among individuals with insufficient sleep, average sleep time was not significantly different among individuals with migraine, those with non-migraine headache, and non-headache $(6.6 \pm 1.2$ vs. $6.5 \pm 1.3$ vs. $6.6 \pm 1.1 \mathrm{~h}$, $p=0.875$ ). Among individuals without insufficient sleep, average sleep time of migraineurs was longer than those with non-headache $(7.9 \pm 1.2$ vs. $7.6 \pm 1.1 \mathrm{~h}, p=0.029)$. However, average sleep time of individuals with migraine did not significantly differ from that of individuals with non-migraine headache $(7.9 \pm 1.2$ vs. $7.5 \pm 1.2 \mathrm{~h}, p=$ 0.109).

Prevalence of insufficient sleep was analyzed according to headache frequency and headache intensity among migraineurs. Prevalence of insufficient sleep in participants with migraineurss with 1-14 attacks per month (44.8\%, $p=0.549)$ and participants with migraine with $\geq 15$ attacks per month $(55.6 \%, p=0.987)$ was not significantly different compared to that of those with $<1$ attack per month (44.7\%). Prevalence of insufficient sleep was not significantly different among migraineurs according to mild, moderate and severe headache intensity $(46.4 \%$ vs. $45.3 \%$ vs. $44.8 \%, p=0.992$ ).

\section{Univariable and multivariable analyses for insufficient sleep}

In univariable analyses, migraine showed an increased OR for insufficient sleep $(\mathrm{OR}=2.4,95 \% \mathrm{CI}=1.7-3.3, p$ $<0.001)$. In addition, short sleep time $(\mathrm{OR}=5.2,95 \% \mathrm{CI}$ $=4.2-6.4, p<0.001)$, insomnia $(\mathrm{OR}=3.9,95 \% \mathrm{CI}=3.0$ $-5.0, p<0.001)$, poor sleep quality $(\mathrm{OR}=3.2,95 \% \mathrm{CI}=$ $2.7-3.9, p<0.001)$, anxiety $(\mathrm{OR}=2.7,95 \% \mathrm{CI}=2.0-$
$3.4, p<0.001)$, and depression $(\mathrm{OR}=2.7,95 \% \mathrm{CI}=1.8-$ $3.9, p<0.001)$ showed increased ORs for insufficient sleep. In multivariable analyses after adjusting for sociodemographic variables (age, gender, size of residential area, educational level and monthly income level; Model 1 ), migraine showed an increased OR for insufficient sleep $(\mathrm{OR}=2.3,95 \% \mathrm{CI}=1.6-3.1, p<0.001)$. In Model 2 after adjusting for sociodemographic variables, poor sleep quality, short sleep time, insomnia, and poor sleep quality $(\mathrm{OR}=1.9,95 \% \mathrm{CI}=1.3-2.8, p=0.001)$ and Model 3 after adjusting for sociodemographic variables, anxiety, and depression $(\mathrm{OR}=1.8,95 \% \mathrm{CI}=1.3-2.6, p$ $=0.001$ ), migraine maintained increased ORs for insufficient sleep. After adjusting for sociodemographic variables, short sleep time, insomnia, poor sleep quality, anxiety, and depression (Model 4), migraine was still a significant factor for insufficient sleep $(\mathrm{OR}=1.8,95 \% \mathrm{CI}$ $=1.2-2.7, p=0.002$, Table 3 ).

\section{Discussion}

The key findings of this study were: (1) The prevalence of insufficient sleep and migraine were $27.0 \%$ and $5.3 \%$, respectively; (2) Although average sleep time was not significantly different according to headache type, sleep need of migraineurs was longer than that of individuals with non-migraine headache or non-headache; (3) The prevalence of insufficient sleep was significantly higher in migraineurs $(45.5 \%)$ compared to that in those with non-migraine headache $(32.9 \%)$ or nonheadache (20.4\%).

This study demonstrated that migraineurs more frequently had insufficient sleep than individuals with nonmigraine headache or non-headache. Enough sleep time and good sleep quality are essential for sufficient sleep $[13,26]$. Sleep time did not differ according to headache type in the present study. Therefore, poor sleep quality

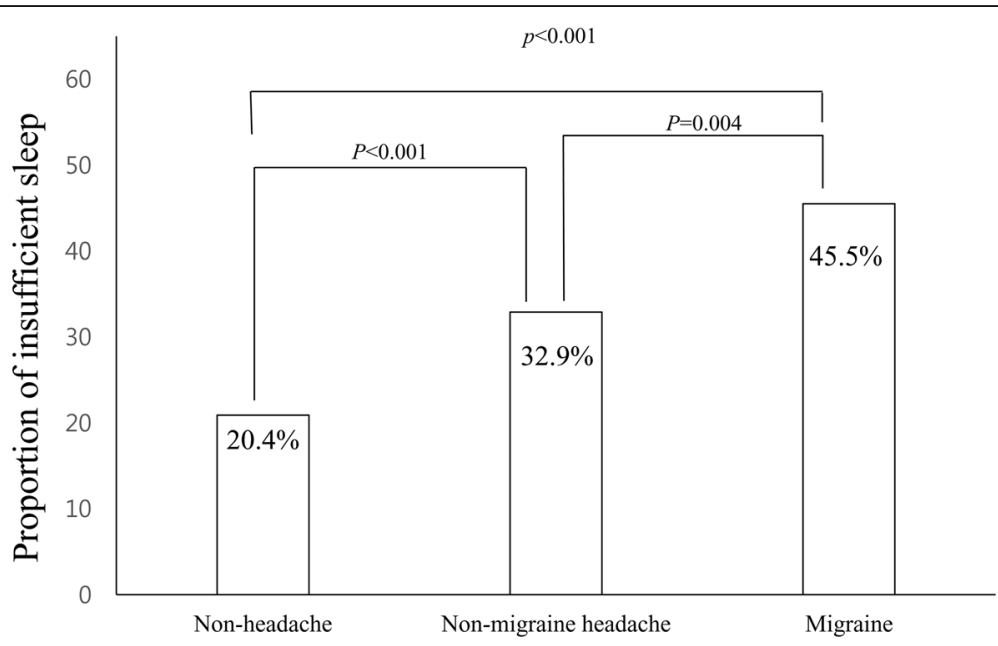

Fig. 2 Prevalence of insufficient sleep among participants with migraine, those with non-migraine headache and non-headache 
Table 3 Univariable and multivariable analyses for insufficient sleep after adjusting for sociodemographics, short sleep time, insomnia, poor sleep quality, anxiety, and depression

\begin{tabular}{|c|c|c|c|c|c|}
\hline & \multirow{2}{*}{$\begin{array}{l}\text { Univariable analysis } \\
\text { OR }(95 \% \mathrm{Cl})\end{array}$} & \multicolumn{4}{|l|}{ Multivariable analysis } \\
\hline & & $\begin{array}{l}\text { Model } 1 \\
\text { OR }(95 \% \mathrm{Cl})\end{array}$ & $\begin{array}{l}\text { Model } 2 \\
\text { OR }(95 \% \mathrm{Cl})\end{array}$ & $\begin{array}{l}\text { Model } 3 \\
\text { OR }(95 \% \mathrm{Cl})\end{array}$ & $\begin{array}{l}\text { Model } 4 \\
\text { OR }(95 \% \mathrm{Cl})\end{array}$ \\
\hline Migraine & $2.4(1.7-3.3), p<0.001$ & $2.3(1.6-3.3), p<0.001$ & $1.9(1.3-2.8), p=0.001$ & $1.8(1.3-2.6), p=0.001$ & $1.8(1.2-2.7), p=0.002$ \\
\hline Short sleep time & $5.2(4.2-6.4), p<0.001$ & & $4.5(3.5-5.7), p<0.001$ & & $4.5(3.6-5.8), p<0.001$ \\
\hline Insomnia & $3.9(3.0-5.0), P<0.001$ & & $2.6(1.9-3.5), P<0.001$ & & $2.4(1.8-3.4), p<0.001$ \\
\hline Poor sleep quality & $3.2(2.7-3.9), p<0.001$ & & $1.6(1.3-2.1), p<0.001$ & & $1.6(1.3-2.0) p<0.001$ \\
\hline Anxiety & $2.7(2.0-3.4), p<0.001$ & & & $2.4(1.8-3.1), p<0.001$ & $1.6(1.2-2.2) p=0.004$ \\
\hline Depression & $2.7(1.8-3.9), p<0.001$ & & & $1.6(1.1-2.5), p=0.022$ & $0.8(0.5-1.3), p=0.321$ \\
\hline
\end{tabular}

Model 1 adjusted for sociodemographics (age, gender, size of residential area, education level and monthly income level)

Model 2 adjusted for sociodemographics, short sleep time, insomnia and poor sleep quality

Model 3 adjusted for sociodemographics, anxiety, and depression

Model 4 adjusted for sociodemographics, short sleep time, insomnia,poor sleep quality, anxiety, and depression

OR Odds Ratio, $\mathrm{Cl}$ Confidence Interval

with similar sleep time among migraineurs might have resulted in insufficient sleep. We also observed frequent poor sleep quality among migraineurs in the present study.

In multivariable logistic regression analyses for insufficient sleep in our study, migraine increased the OR for insufficient sleep even after adjusting for sociodemographic variables, short sleep time, insomnia, poor sleep quality, anxiety, and depression. This finding might be contradictory to our assumption that insufficient sleep among migraineurs resulted from poor sleep quality. One possible explanation for the discrepancy was that insufficient sleep among migraineurs was not only due to poor sleep quality, but also due to the fact that migraine itself was associated with insufficient sleep. Another possible explanation might be due to sleep quality assessment instrument. We used PSQI to assessing sleep quality in the present study. Although PSQI is a widely using instrument for assessing sleep quality, PSQI might not properly reflect sleep quality of migraineurs. Further studies regarding sleep quality among migraineurs using other validated sleep quality assessing instruments are needed.

In the present study, migraineurs had more sleep need compared to individuals with non-migraine headache and non-headache. One possible explanation for increased sleep need among migraineurs is migraineurs' attempt to sleep for relieving migraine attacks. Migraineurs often report relieving migraine symptoms by sleep [27, 28]. Therefore, migraineurs may need longer sleep time than non-migraineurs for relieving their migraine attacks. Another possible explanation for increased sleep need among migraineurs is their decreased sleep quality. Decreased sleep quality among migraineurs has been repeatedly reported [7, 8]. Accordingly, migraineurs need more sleep for sufficient sleep.
The demonstration of insufficient sleep among migraineurs in the present study was compatible with previous electrophysiological studies regarding the relationship between sleep and migraine [29,30]. A case-controlled study including 53 migraineurs and 34 controls has reported that migraineurs have higher awaking index, strong tendency for more slow wave sleep, and lower fast arousal index during interictal period [29]. In addition, migraineurs have less sleep latency compared to controls during the preictal period. These findings have been interpreted as migraineurs commonly suffer from relative sleep deprivation and need more sleep than healthy controls [29]. Another case-controlled study has revealed that non-sleep-related migraine patients have more slow wave sleep and higher K-burst index compared to sleep-related migraine patients or controls, indicating more sleep deprivation [30].

The overall prevalence of insufficient sleep was $27.0 \%$ in present study. This prevalence was similar to that reported in previous studies [14, 31, 32]. A survey in Finland has revealed that the prevalence of insufficient sleep is $20.4 \%$ among those aged 33 to 60 years [14]. A telephone survey in Australia has shown that insufficient sleep prevalence is $28 \%$ [31]. A Swedish population survey for people aged 30 to 65 years has revealed that $28 \%$ of females and $21 \%$ of males have experienced too little sleep [32].

In present study, the 1-year prevalence of migraine $(5.3 \%)$ is lower than that of European (10-25\%) and North American (9-16\%) studies [33]. The 1-year prevalence of migraine in Asian countries has been found to range between $4.7 \%$ and $9.1 \%$ in most studies [19, 34] Therefore, the 1-year prevalence of migraine in the present study was similar to that in Asian countries. Similarity in the prevalence of insufficient sleep and migraine between our study and previous studies suggested that insufficient sleep and migraine were properly evaluated in the present study. 
The overall response rate in the present study was not high. However, we used two-stage clustered random sampling, proportional to the population distribution of Korea. Therefore, distribution of age, sex, size of residential area, educational level and monthly income level of our participants was not different from those of Korean general population. In addition, the prevalence of migraine, anxiety, depression, poor sleep quality and insomnia in the data of KHSS were similar to that in previous population-based studies $[16,35,36]$ Therefore, we could assure that we successfully investigated migraine and insufficient sleep in the present study.

This study has some limitations. First, sleep parameters such as total sleep time and sleep quality were not evaluated by objective methods such as polysomnography (PSG). We evaluated sleep habits and sleep quality with self-reported questionnaires. In population based study, it is practically difficulty to perform PSG to evaluate sleep parameters and sleep quality in all participants. Second, although the present study was a large population-based study, some subgroup analyses might not have statistical significance due to the limited sample size. In other words, the lack of significant findings in subgroup analyses could be due to limited sample size.

The present study also has some strength. First, the present study had a large sample size. The distribution of age, gender, size of residential area, education level and monthly income level in participants represented the Korean general population. Second, this study evaluated covariates such as sociodemographic variables, short sleep time, insomnia, poor sleep quality, anxiety, and depression which could affect insufficient sleep. The relationship between migraine and insufficient sleep was significantly maintained after adjusting for covariates.

\section{Conclusion}

Approximately half of migraineurs experienced insufficient sleep. The prevalence of insufficient sleep was higher in individuals with migraine compared to that in those with non-migraine headache or non-headache. Although the average sleep time was not significantly different among individuals with migraine, those with nonmigraine headache, and non-headache, sleep need was longer among migraineurs than that among individuals with non-migraine or non-headache. Our findings suggest that migraineurs need longer sleep time than individuals with non-migraine or non-headache to have sufficient sleep.

\section{Abbreviations}

Cl: Confidence interval; GAS: Goldberg anxiety scale; ICHD: International classification of headache disorders; ISI: Insomnia severity index; KHSS: Korean headache-sleep study; OR: Odds ratio; PHQ-9: Patient health questionnaire-9; PSG: Polysomnography; PSQI: Pittsburgh sleep quality index score

\section{Acknowledgements}

The authors would like to thank Gallup Korea for providing technical support for the Korean Headache-Sleep Study.

Funding

This Study was Supported by a 2011-Grant from Korean Academy of Medical Sciences.

\section{Authors' Contributions}

JYK conceptualized and designed the study, analyzed the data and wrote the manuscript. SJC, WJK, KIY and CHY conceptualized and collected the data. MKC conceptualized and designed the study, collected and analyzed the data, and wrote the manuscript. All authors read and approved the final manuscript.

\section{Competing Interests}

The authors declare that they have no competing interests.

\section{Publisher's Note}

Springer Nature remains neutral with regard to jurisdictional claims in published maps and institutional affiliations.

\section{Author details}

'Department of Neurology, Bio Medical Research Institute, Pusan Nationa University Hospital, Pusan National University School of Medicine, Busan, South Korea. ${ }^{2}$ Department of Neurology, Dongtan Sacred Heart Hospital, Hallym University College of Medicine, Hwaseong, South Korea. ${ }^{3}$ Department of Neurology, Gangnam Severance Hospital, Yonsei University College of Medicine, Seoul, South Korea. ${ }^{4}$ Sleep Disorders Center, Department of Neurology, Soonchunhyang University College of Medicine, Cheonan Hospital, Cheonan, South Korea. ${ }^{5}$ Department of Neurology, Bundang Clinical Neuroscience Institute, Seoul National University Bundang Hospital, Seongnam, South Korea. ${ }^{6}$ Department of Neurology, Kangnam Sacred Heart Hospital, Hallym University College of Medicine, Seoul, South Korea.

Received: 20 February 2017 Accepted: 20 April 2017

Published online: 28 April 2017

\section{References}

1. Odegard SS, Engstrom M, Sand T, Stovner L, Zwart JA, Hagen K (2010) Associations between sleep disturbance and primary headaches: the third nord-trondelag health study. J Headache Pain 11(3):197-206

2. Kelman L, Rains JC (2005) Headache and sleep: examination of sleep patterns and complaints in a large clinical sample of migraineurs. Headache 45(7):904-910

3. Breslau N, Davis GC, Schultz LR, Peterson EL (1994) Joint 1994 Wolff award presentation. Migraine and major depression: a longitudinal study. Headache 34(7):387-393

4. Hauge AW, Kirchmann M, Olesen J (2010) Trigger factors in migraine with aura. Cephalalgia 30(3):346-353

5. Andress-Rothrock D, King W, Rothrock J (2010) An analysis of migraine triggers in a clinic-based population. Headache 50(8):1366-1370

6. Scher Al, Lipton RB, Stewart WF (2003) Habitual snoring as a risk factor for chronic daily headache. Neurology 60(8):1366-1368

7. Qiu C, Frederick IO, Sorensen T, Aurora SK, Gelaye B, Enquobahrie DA, Williams MA (2015) Sleep disturbances among pregnant women with history of migraines: a cross-sectional study. Cephalalgia 35(12):1092-1102

8. Zhu Z, Fan X, Li X, Tan G, Chen L, Zhou J (2013) Prevalence and predictive factors for poor sleep quality among migraineurs in a tertiary hospital headache clinic. Acta Neurol Belg 113(3):229-235

9. Schoonman GG, Evers DJ, Terwindt GM, van Dijk JG, Ferrari MD (2006) The prevalence of premonitory symptoms in migraine: a questionnaire study in 461 patients. Cephalalgia 26(10):1209-1213

10. Giffin NJ, Ruggiero L, Lipton RB, Silberstein SD, Tvedskov JF, Olesen J, Altman J, Goadsby PJ, Macrae A (2003) Premonitory symptoms in migraine: an electronic diary study. Neurology 60(6):935-940

11. Maia Q, Grandner MA, Findley J, Gurubhagavatula I (2013) Short and long sleep duration and risk of drowsy driving and the role of subjective sleep insufficiency. Accid Anal Prev 59:618-622

12. Strine TW, Chapman DP (2005) Associations of frequent sleep insufficiency with health-related quality of life and health behaviors. Sleep Med 6(1):23-27 
13. Altman NG, Izci-Balserak B, Schopfer E, Jackson N, Rattanaumpawan P, Gehrman PR, Patel NP, Grandner MA (2012) Sleep duration versus sleep insufficiency as predictors of cardiometabolic health outcomes. Sleep Med 13(10):1261-1270

14. Hublin C, Kaprio J, Partinen M, Koskenvuo M (2001) Insufficient sleep-a population-based study in adults. Sleep 24(4):392-400

15. Holt JB, Zhang X, Sizov N, Croft JB (2015) Airport noise and self-reported sleep insufficiency, United States, 2008 and 2009. Prev Chronic Dis 12, E49

16. Cho SJ, Chung YK, Kim JM, Chu MK (2015) Migraine and restless legs syndrome are associated in adults under age fifty but not in adults over fifty: a population-based study. J Headache Pain 16:554

17. Headache Classification Subcommittee of the International Headache $S$ (2004) The international classification of headache disorders: 2 nd edition. Cephalalgia 24 Suppl 1:9-160

18. Stang PE, Osterhaus JT (1993) Impact of migraine in the United States: data from the national health interview survey. Headache 33(1):29-35

19. Kim BK, Chu MK, Lee TG, Kim JM, Chung CS, Lee KS (2012) Prevalence and impact of migraine and tension-type headache in Korea. J Clin Neurol 8(3): 204-211

20. Morin CM, Belleville G, Belanger L, Ivers H (2011) The insomnia severity index: psychometric indicators to detect insomnia cases and evaluate treatment response. Sleep 34(5):601-608

21. Goldberg D, Bridges K, Duncan-Jones P, Grayson D (1988) Detecting anxiety and depression in general medical settings. BMJ 297(6653):897-899

22. Lim JY, Lee SH, Cha YS, Park HS, Sunwoo S (2001) Reliability and validity of anxiety screening scale. J Korean Acad Fam Med 22(8):1224-1232

23. Kim JS, Kim YS, Lee GY, Park TJ, Lee YH, Kong BK, Sim JC (1997) The standardization of Korean-translated Goldberg's shart screening scale for anxiety and depression. J Korean Acad Fam Med 18(12):1452-1460

24. Pignone MP, Gaynes BN, Rushton JL, Burchell CM, Orleans $C T$, Mulrow CD, Lohr KN (2002) Screening for depression in adults: a summary of the evidence for the U.S. Preventive services task force. Ann Intern Med 136(10): 765-776

25. Choi HS, Choi JH, Park KH, Joo KJ, Ga H, Ko HJ, Kim SR (2007) Standardization of the Korean version of patient health questionnaire-9 as a screening instrument for major depressive disorder. J Korean Acad Fam Med 28(2):114-119

26. Pilcher JJ, Ginter DR, Sadowsky B (1997) Sleep quality versus sleep quantity: relationships between sleep and measures of health, well-being and sleepiness in college students. J Psychosom Res 42(6):583-596

27. Haque B, Rahman KM, Hoque A, Hasan AT, Chowdhury RN, Khan SU, Alam MB, Habib M, Mohammad QD (2012) Precipitating and relieving factors of migraine versus tension type headache. BMC Neurol 12:82

28. Bag B, Karabulut N (2005) Pain-relieving factors in migraine and tensiontype headache. Int J Clin Pract 59(7):760-763

29. Engstrom M, Hagen K, Bjork MH, Stovner LJ, Gravdahl GB, Stjern M, Sand T (2013) Sleep quality, arousal and pain thresholds in migraineurs: a blinded controlled polysomnographic study. J Headache Pain 14:12

30. Engstrom M, Hagen K, Bjork M, Gravdahl GB, Sand T (2013) Sleep-related and non-sleep-related migraine: interictal sleep quality, arousals and pain thresholds. J Headache Pain 14:68

31. Lack L, Miller W, Turner D (1988) A survey of sleeping difficulties in an Australian population. Community Health Stud 12(2):200-207

32. Liljenberg B, Almqvist M, Hetta J, Roos BE, Agren H (1988) The prevalence of insomnia: the importance of operationally defined criteria. Ann Clin Res 20(6):393-398

33. Stovner L, Hagen K, Jensen R, Katsarava Z, Lipton R, Scher A, Steiner T, Zwart JA (2007) The global burden of headache: a documentation of headache prevalence and disability worldwide. Cephalalgia 27(3):193-210

34. Peng KP, Wang SJ (2014) Epidemiology of headache disorders in the Asiapacific region. Headache 54(4):610-618

35. Song TJ, Cho SJ, Kim WJ, Yang KI, Yun CH, Chu MK (2016) Anxiety and depression in probable migraine: a population-based study, Cephalalgia. [Epub ahead of print]

36. Oh K, Cho SJ, Chung YK, Kim JM, Chu MK (2014) Combination of anxiety and depression is associated with an increased headache frequency in migraineurs: a population-based study. BMC Neurol 14:238

\section{Submit your manuscript to a SpringerOpen ${ }^{\circ}$ journal and benefit from:}

- Convenient online submission

- Rigorous peer review

- Immediate publication on acceptance

- Open access: articles freely available online

- High visibility within the field

- Retaining the copyright to your article

Submit your next manuscript at $\gg$ springeropen.com 